(2) Open Access Full Text Article

ORIGINAL RESEARCH

\title{
Preparation and in vitro/in vivo evaluation of PLGA microspheres containing norquetiapine for long-acting injection
}

This article was published in the following Dove Press journal:

Drug Design, Development and Therapy

\author{
Chun-Woong Park' \\ Hyo-Jung Lee' \\ Dong-Won Oh' \\ Ji-Hyun Kang' \\ Chang-Soo Han' \\ Dong-Wook Kim²
}

'College of Pharmacy, Chungbuk National University, Cheongju, Republic of Korea; ${ }^{2}$ Department of Pharmaceutical Engineering, Cheongju University, Cheongju, Republic of Korea
Correspondence: Dong-Wook Kim Department of Pharmaceutical Engineering, Cheongju University, Daesung-ro 298, Cheongju 28530, Republic of Korea

Tel +82 432297984

Email pharmengin@gmail.com
Background: Norquetiapine ( $N$-desalkyl quetiapine, NQ) is an active metabolite of quetiapine with stable pharmacokinetic and pharmacological properties. However, its short half-life is a drawback for clinical applications, and long-acting formulations are required.

Purpose: The objectives of this study were to prepare improved entrapment efficiency NQ freebase microspheres by the solvent evaporation method with poly(d,l-lactic-co-glycolic acid) (PLGA) as a release modulator and to evaluate their physicochemical and in vitro/in vivo release properties.

Methods: NQ freebase PLGA (1:5 w/w) formulations were prepared by the oil-in-water $(\mathrm{o} / \mathrm{w})$ emulsion-solvent evaporation method. A solution of the drug and PLGA in 9:1 v/v dichloromethane:ethanol was mixed with $0.2 \%$ polyvinyl alcohol and homogenized at 2,800 rpm. The emulsion was stirred for $3 \mathrm{~h}$ to dilute and evaporate the solvent. After that, the resulting product was freeze-dried. Drug-loading capacity was measured by the validated RP-HPLC method. The surface morphology of the microspheres was observed by scanning electron microscopy (SEM), and the physicochemical properties were evaluated by differential scanning calorimetry, powder X-ray diffraction, and Fourier-transform infrared spectroscopy particle size distribution. The in vitro dissolution test was performed using a rotary shaking bath at $37^{\circ} \mathrm{C}$, with constant shaking at $50 \mathrm{rpm}$ in sink condition.

Results: The NQ freebase microspheres prepared by o/w emulsion-solvent evaporation showed over $30 \%$ efficiency. NQ was confirmed to be amorphous in the microspheres by powder $\mathrm{X}$-ray diffraction and differential scanning calorimetry. Special chemical interaction in the microspheres was not observed by FT-IR. The in vitro dissolution test demonstrated that the prepared microspheres' release properties were maintained for more than 20 days. The in vivo test also confirmed that the particles' long acting properties were maintained. Therefore, good in vitro-in vivo correlation was established.

Conclusion: In this study, NQ freebase-PLGA microspheres showed potential for the treatment of schizophrenia for long-periods.

Keywords: schizophrenia, freebase, o/w emulsion-solvent evaporation, freeze dry, active metabolite, IVIVC

\section{Introduction}

Schizophrenia is defined by the National Institutes of Health (NIH) as a chronic and severe mental disorder that affects how a person thinks, feels, and behaves. Treatment is long term and often subjected to inconsistent dosing regimens, which increases the risk of relapse. High doses and fluctuating drug plasma concentrations associated with ordinary daily treatment may also cause tardive dyskinesia. ${ }^{1}$ Schizophrenia is commonly treated with oral antipsychotics, due to ease of administration and noninvasiveness of 
therapy; however, the frequency of medication results in low patient compliance, which limits the scope of schizophrenia treatment. ${ }^{2}$ A long-acting formulation that maintains consistent drug concentration in plasma and that delivers the lowest effective dose could both prevent the recurrences of psychosis and lower the risk of side effects such as tardive dyskinesia. ${ }^{3}$

$N$-desalkyl quetiapine (or norquetiapine, NQ) is a major active metabolite of quetiapine in humans, produced by the isoenzyme CYP3A4 in cytochrome P450. ${ }^{20}$ Quetiapine was originally developed as a second-generation antipsychotic agent for the treatment of schizophrenia. ${ }^{4}$ The body recognizes quetiapine as a xenobiotic or a foreign agent and metabolizes it into several compounds, including active metabolite. An active metabolite is a substance that has therapeutic activity. ${ }^{5}$ Quetiapine has been reported to have pharmacokinetic variability among older adults and in patients using concomitant medications. ${ }^{6}$ Inductors such as carbamazepine and phenytoin enhance the activity of quetiapine, leading to the formation of NQ, whereas inhibitors such as ketoconazole, itraconazole, erythromycin, and fluvoxamine suppress the activity of quetiapine. $^{7}$ Norquetiapine, an active metabolite of quetiapine, is less affected by differences in the degree of metabolism among individuals, such as age, and by other drugs, and it has therapeutic effect. Norquetiapine inhibits the noradrenaline transporter and has partial agonistic activity at 5- $\mathrm{HT}_{1 \mathrm{~A}}$ receptors. ${ }^{8}$ Activity at both these targets is hypothesized to contribute to antidepressant activity. Norquetiapine has a 9-12 h half-life in humans. ${ }^{9}$ For maintaining constant blood concentration for better schizophrenia treatment, a long-acting formulation of norquetiapine is required. Many antipsychotics have been studied as injectable long-acting formulations. The first injectable long-acting antipsychotic formulation was developed in the 1960s. Currently, some antipsychotic drugs are being developed in a similar manner, such as Abilify Maintena (aripiprazole monohydrate), Zyprexa Relprevv (olanzapine pamoate), and Invega Trinza (paliperidone palmitate), which are available in the US and in the UK. ${ }^{10}$ Long-acting antipsychotic formulations can avoid the first-pass metabolism and there is certainty of delivery of the therapeutic agents. Further, the long-acting effect is expected to mitigate non-adherence and reduce the risk of relapse in comparison to the oral administration that has low patient compliance. ${ }^{11}$ The number of days of hospitalization for patients can be reduced. Poly(D,L-lactic-co-glycolic acid) (PLGA) polymers are widely used for controlled delivery of drugs via parenteral route. They have been approved as safe for the human body by the US Food and Drug Administration (FDA) with good biodegradability and biocompatibility. ${ }^{12}$ Many studies on the PLGA formulation have been conducted, ensuring sustained release of therapeutic agents, ranging from a few weeks to several months. PLGA microspheres with atypical antipsychotics reduced the dosing frequency, leading to measurable increase in adherence to treatment regimens in a schizophrenic patient population. ${ }^{13}$ The NQ freebase PLGA $502 \mathrm{H}$ formulation was chosen to investigate the physiochemical characteristics, in vitro dissolution, in vivo release, and in vitro-in vivo correlation (IVIVC). The aims of this study were to develop long-acting microspheres using PLGA with the advantages of norquetiapine as an active metabolite and to evaluate their long-term release properties for better treatment, continuous management of disease, and patient compliance.

\section{Materials and methods Materials}

Norquetiapine dihydrochloride (NQ.2HCl) was purchased from Toronto Research Chemicals, Ltd. (Toronto, Canada) and used for the synthesis of norquetiapine freebase (NQ freebase). Poly(D,L-lactic-co-glycolic acid) and PLGA 502, 502H, 503, and 503H were supplied by Evonik, Ltd. (Essen, Germany). Polyvinyl alcohol 500 (PVA 500) was obtained from OCI Company, Ltd. (Seoul, Republic of Korea). Methanol, dichloromethane, and acetonitrile were of highperformance liquid chromatography (HPLC) grade and were purchased from Honeywell Burdick \& Jackson Ltd. (Muskegon, MI, USA). Anhydrous dibasic sodium phosphate was purchased from Georgiachem (Suwanee, GA, USA). Potassium phosphate monobasic was purchased from Daejung Ltd. (Siheung, Republic of Korea). All experiments were carried out using Milli- $\mathrm{Q}^{\circledR}$ distilled water.

\section{Preparation of NQ freebase}

One gram of $\mathrm{NQ} \cdot 2 \mathrm{HCl}$ was dissolved completely in $5 \mathrm{~mL}$ of $1 \mathrm{~N}$ sodium carbonate solution and stirred at $24^{\circ} \mathrm{C}-27^{\circ} \mathrm{C}$ for $30 \mathrm{~min}$. The synthetic product obtained was extracted using methylene chloride. Then, the product was filtered and dried. A total of $0.89 \mathrm{~g}$ of NQ freebase was obtained.

\section{Preparation of PLGA microspheres with NQ freebase}

NQ PLGA microspheres were prepared by oil-in-water (o/w) emulsion-solvent evaporation. ${ }^{14}$ For each PLGA formulation (PLGA 502, PLGA 502H, PLGA 503, and PLGA 503H), $300 \mathrm{mg}$ was dissolved along with $60 \mathrm{mg}$ NQ freebase in $15 \mathrm{~mL}$ dichloromethane:methanol solution $(9: 1 \mathrm{v} / \mathrm{v})$ and injected into $300 \mathrm{~mL} 0.2 \%$ polyvinyl alcohol at $1 \mathrm{~mL} / \mathrm{s}$ with a highspeed dispersion homogenizer (S18N-19G; IKA, Staufen, Germany) at 2,800 rpm for $1 \mathrm{~min}$ to form an o/w emulsion. ${ }^{15}$ The emulsion was stirred for $3 \mathrm{~h}$ at $24^{\circ} \mathrm{C}-27^{\circ} \mathrm{C}$ for dilution and 
evaporation of the solvent. The microspheres were centrifuged at 1,500 rpm for $3 \mathrm{~min}$ and washed three times with distilled water before being collected using a $0.45 \mu \mathrm{m}$ filter and distributed with $5 \mathrm{~mL}$ distilled water. Finally, the microspheres were pretreated and lyophilized for two days at $-75^{\circ} \mathrm{C}$.

\section{Morphology and particle size}

The scanning electron microscopic (SEM) images of NQ freebase PLGA 502H microspheres were obtained using GEMINI LEO $1530^{\circledR}$ (Zeiss Ltd., Jena, Germany), as the dissolution patterns were appropriate for the desired longlasting effect. Samples were mounted on an aluminum plate using a carbon tape, then placed inside a Hummer VI sputtering device (Minneapolis, MN, USA), and coated with platinum to discharge the particles. The particle size of the NQ freebase PLGA 502H microspheres was measured with image analysis software (NIH, ImageJ).

\section{Determination of drug-loading efficiency and encapsulation efficiency}

The entrapped amount of drug in the NQ freebase PLGA $502 \mathrm{H}$ microspheres was measured by HPLC. Five milligram of NQ freebase PLGA 502H microspheres was dissolved in $50 \mathrm{~mL}$ of $60 \%$ acetonitrile solution using Branson Model $8510^{\circledR}$ Ultrasonic Cleaner (BRANSON Ltd., St Louis, MO, USA) for $30 \mathrm{~min}$. Analysis was performed using Ultimate $3000^{\circledR}$ HPLC system (Thermo Fisher Scientific, Waltham, MA, USA) and $5 \mu \mathrm{m} \mathrm{C8}$ column $(100 \mathrm{~A} 250 \times 4.6 \mathrm{~mm})$ from Phenomenex Ltd. (Torrance, CA, USA). The mobile phase was delivered at a flow rate of $1.5 \mathrm{~mL} / \mathrm{min}$, the detection wavelength was set at $225 \mathrm{~nm}$, and the injection volume was $20 \mu \mathrm{L}$. The drug-loading efficiency (\%) and entrapment efficiency ( $\%$ ) were calculated using the following equations (Equations 1 and 2), respectively. All measurements were conducted in triplicate.

Drug loading $(\%)=$

$$
\frac{\text { Amount of drug in microspheres }}{\text { Volume of microspheres }} \times 100
$$

Entrapment efficiency $(\%)=$ $\frac{\text { Amount of drug in microspheres }}{\text { Theoretical amount of drug }} \times 100$

\section{Differential scanning calorimetry (DSC)}

DSC was performed using Q2000 ${ }^{\circledR}$ (TA Instruments Ltd., New Castle, DE, USA). One milligram of the prepared microspheres was placed on aluminum pan and hermetically sealed. The samples were heated from $0^{\circ} \mathrm{C}$ to $300^{\circ} \mathrm{C}$ at a rate of $20^{\circ} \mathrm{C} / \mathrm{min}$ under a nitrogen flow of $50 \mathrm{~mL} / \mathrm{min}$.

\section{Powder X-ray diffraction (PXRD)}

PXRD patterns were obtained using X'Pert PRO MRD ${ }^{\circledR}$ (PANalytical Ltd., Almelo, the Netherlands) with $\mathrm{Cu} \mathrm{K \alpha}$ radiation generated at $200 \mathrm{~mA}$ and $45 \mathrm{kV}$. The samples were placed on a silicon plate at room temperature and $2 \theta$ scans were collected from $5^{\circ}$ to $60^{\circ}$.

\section{Fourier-transform infrared spectroscopy (FT-IR)}

Infrared spectroscopy was performed on the prepared microspheres in the range of 600 to $4,000 \mathrm{~cm}^{-1}$, using FT/IR-4100 type A (Jasco Corporation, Tokyo, Japan).

\section{In vitro release study}

A total of $10 \mathrm{mg}$ of NQ freebase PLGA microspheres was suspended in $10 \mathrm{~mL}$ of phosphate-buffered saline (PBS, $\mathrm{pH}=7.4)$ and incubated at $37^{\circ} \mathrm{C} \pm 0.5^{\circ} \mathrm{C}$ in a rotary shaking incubator (C-SKI-2; Chang-Sing Science, Seoul, Republic of Korea) at $50 \mathrm{rpm}$ for 20 days. At each predetermined sampling point, the supernatant was separated for a validated RP-HPLC assay, and fresh medium of equal volume was added. All assays were performed in triplicate. ${ }^{16}$

\section{In vivo pharmacokinetic study}

Animals were treated and maintained in accordance with the principles of laboratory animal care and approved by the Committee for Animal Experiments of Chungbuk National University (Cheongju, Republic of Korea). Sprague Dawley rats (male, 400-460 g) were purchased from Daehan Biolink Co. (Eumseong, Republic of Korea) and fed ad libitum. The rats were randomly divided into two groups. One group $(n=6)$ received intramuscular (IM) injections of NQ freebase $30 \%$ ethanol solution $(5.4 \mu \mathrm{g} / 0.2 \mathrm{~mL})$, chosen due to the poor solubility of the NQ freebase in water. The other group $(n=6)$ was administered NQ freebase PLGA 502H microspheres (corresponding to $162 \mu \mathrm{g} \mathrm{NQ} / 0.2 \mathrm{~mL}$ ) intramuscularly. At each predetermined sampling point, blood samples were collected for liquid chromatography-mass spectrometry assay.

\section{IVIVC}

IVIVC is a predictive model that is used to describe the relationship between the in vitro behavior of formulation and relevant in vivo profiles. ${ }^{17}$ Level A correlation which is the most informative and recommended by the FDA is a point-to-point relationship in vitro and in vivo. That is a point-to-point relationship in vitro and in vivo. For applying 
Table I Solubility of norquetiapine dihydrochloride and norquetiapine freebase

\begin{tabular}{lll}
\hline Property & NQ.2HCl & NQ freebase \\
\hline PKa & $3.93,8.49$ & $3.90,8.55$ \\
MW $(\mathrm{g} / \mathrm{mol})$ & 368.32 & 295.40 \\
Solubility $(\mu \mathrm{g} / \mathrm{mL})$ & 325.78 & 6.82 \\
Log P & 3.12 & 3.18 \\
\hline
\end{tabular}

Abbreviations: MW, molecular weight; NQ, N-desalkyl quetiapine.

level A correlation, same time points were chosen. Fraction of absorption was obtained by deconvolution using the Wagner-Nelson method. ${ }^{18}$

\section{Results and discussion Preparation of NQ freebase PLGA 502H microspheres}

NQ freebase was synthesized from NQ·2HCl. The NQ freebase PLGA microspheres were prepared by o/w emulsion, which results in high yield and uniform particles. ${ }^{19}$ In preliminary experiments, PLGA 503 microspheres containing NQ$\cdot 2 \mathrm{HCl}$ were prepared using o/w emulsion solvent evaporation (data not shown). The entrapment efficiency was observed to be less than $0.1 \%$ in the prepared particles, meaning that when the oil phase was mixed with the water phase, the salt form of NQ was almost completely dissolved in the water phase. NQ freebase was synthesized to increase the entrapment efficiency of the drug in the microspheres. ${ }^{20}$ Table 1 shows the pKa, molecular weight, and solubility values of $\mathrm{NQ} \cdot 2 \mathrm{HCl}$ and $\mathrm{NQ}$ freebase as analyzed by using the T3 equipment (Lakewood, NJ, USA). According to the solubility values obtained, $\mathrm{NQ} \cdot 2 \mathrm{HCl}$ had a solubility of $325.78 \mu \mathrm{g} / \mathrm{mL}$ and NQ freebase $6.82 \mu \mathrm{g} / \mathrm{mL}$. The salt form showed about 50 times higher solubility than the drug freebase. NQ freebase microspheres prepared by o/w emulsion solvent evaporation were confirmed to have much higher entrapment efficiency than that of NQ.2HCl in PLGA 503. NQ freebase PLGA microspheres using different PLGA weights (PLGA 502, PLGA 502H, PLGA 503, PLGA 503H) were prepared to identify the correlation between entrapment efficiency and both molecular weight and the presence of acid terminals. The entrapment efficiency and amount of drug loaded in the prepared microspheres are shown in Table 2. The larger molecular weight showed an increase of about 2\%. Acid-terminated non-end-capped PLGA (PLGA 502H and $503 \mathrm{H}$ ) showed about $10 \%$ higher drug efficiency than the same molecular weight PLGA without the acid terminus. This suggests that the acid-terminated part of the polymer interacts with the amine group of the NQ. ${ }^{16}$

\section{Physiochemical characteristics of PLGA $502 \mathrm{H}$ microspheres}

SEM and particle size distribution

The SEM images of NQ.2HCl, NQ freebase, PLGA 502, PLGA 502H, and NQ freebase PLGA 502H microspheres are shown in Figure 1. NQ.2HCl was shown to have an angular, crystalline form (Figure 1A). NQ freebase microspheres (Figure 1B) grew larger and developed a round shape during synthesis. PLGA 502 and PLGA 502H (Figure 1C and D, respectively) both showed a fibrous morphology and no difference in the SEM images. The prepared microspheres did not have the original shape of the drug, and the fibrous nature of the surface polymer was lost. Figure $1 \mathrm{E}$ and $\mathrm{F}$ shows that the microspheres have a non-porous structure, round shape, and a smooth surface with no drug crystals. Large microspheres can cause severe pain when administered intramuscularly. The prepared PLGA 502H microspheres were measured using an image analysis program (NIH, imageJ); they had a diameter of $8.86 \pm 3.11 \mu \mathrm{m}$ that is acceptable for IM injection system. ${ }^{21}$

\section{DSC}

The DSC thermograms of NQ.2HCl, NQ freebase, PLGA $502 \mathrm{H}$, and NQ freebase PLGA 502H microspheres, as well as a physical mixture of the microspheres, were obtained and the results are shown in Figure 2. The DSC result was obtained by raising the temperature to $20^{\circ} \mathrm{C} / \mathrm{min}$. The $\mathrm{T}_{\mathrm{m}}$ of $\mathrm{NQ} \cdot 2 \mathrm{HCl}$ was found to be around $180^{\circ} \mathrm{C}-210^{\circ} \mathrm{C}$, whereas the NQ freebase was found to have a $\mathrm{T}_{\mathrm{g}}$ between $50^{\circ} \mathrm{C}$ and $65^{\circ} \mathrm{C}$. The $\mathrm{T}_{\mathrm{m}}$ of the NQ freebase was not observed. It is likely that the temperature difference was caused by the crystal form changing into the amorphous form upon the removal of the base from NQ.2HCl. A clear $T_{m}$ peak in the NQ freebase was not observed. In the case of PLGA $502 \mathrm{H}$, the $\mathrm{T}_{\mathrm{m}}$ peak

Table 2 Drug efficiency and drug-loading capacity of NQ freebase PLGA 502, PLGA 502H, PLGA 503, and PLGA 503H microspheres

\begin{tabular}{lllll}
\hline Property & NQ freebase & NQ freebase & NQ freebase & NQ freebase \\
& PLGA 502 MSs & PLGA 502H MSs & PLGA 503 MSs & $\begin{array}{l}\text { PLGA 503H } \\
\text { MSs }\end{array}$ \\
\hline Efficiency (\%) & $30.33 \pm 0.51$ & $46.19 \pm 0.33$ & $32.69 \pm 1.28$ & $48.30 \pm 3.04$ \\
Drug-loading capacity (\%) & $5.0 \pm 0.08$ & $7.4 \pm 0.06$ & $5.48 \pm 0.19$ & $8.21 \pm 0.52$ \\
\hline
\end{tabular}

Abbreviations: NQ, N-desalkyl quetiapine; MSs, microspheres; PLGA, poly(D,L-lactic-co-glycolic acid). 

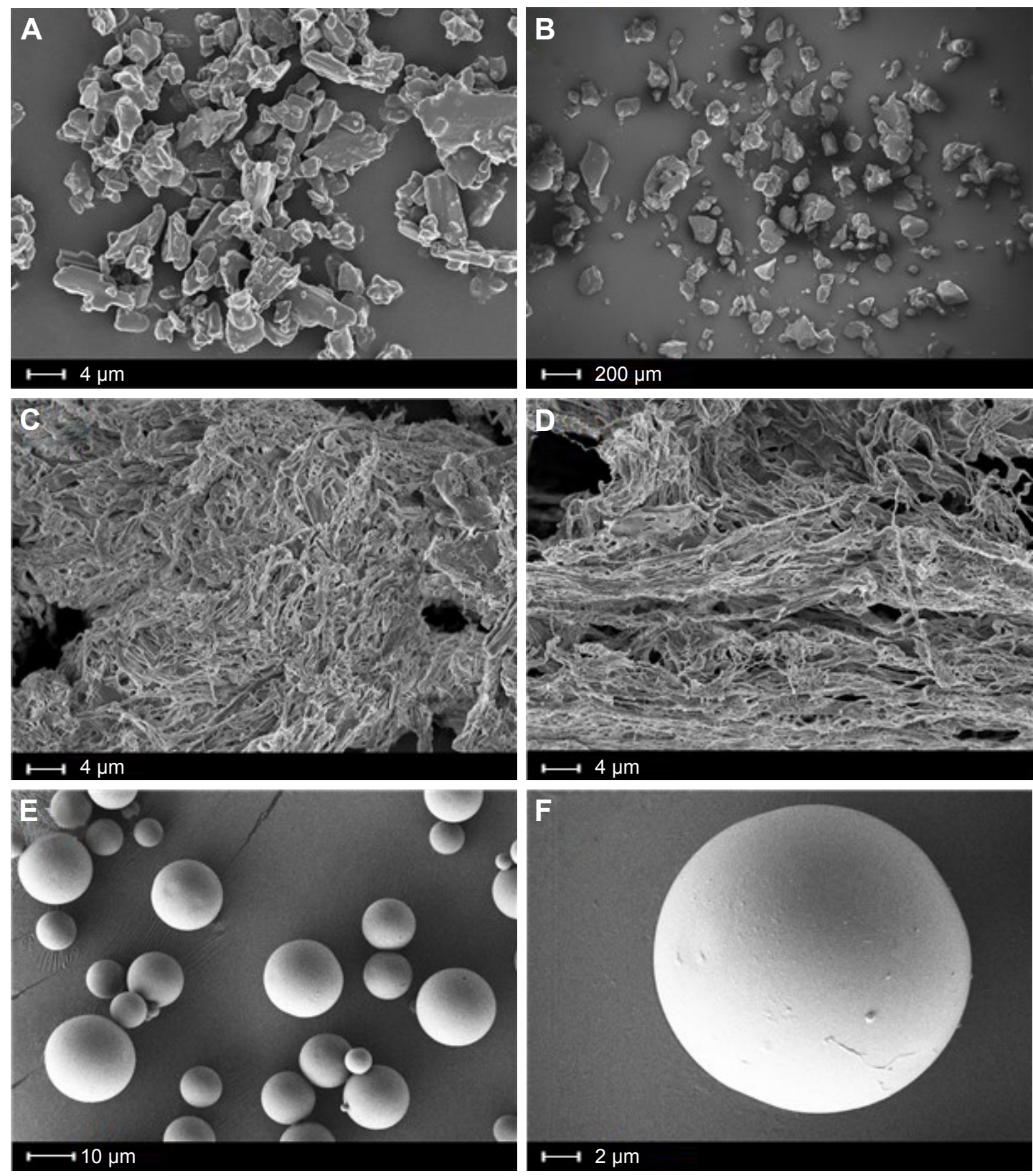

Figure I SEM micrographs of NQ freebase PLGA 502H MSs: (A) NQ.2HCl (5,000×), (B) NQ freebase (I00×), (C) PLGA 502 (5,000×), (D) PLGA 502H (5,000×), and (E and F) NQ freebase PLGA $502 \mathrm{H}$ MSs (2,500× and $10,000 \times$ respectively).

Abbreviations: SEM, scanning electron microscopy; NQ, N-desalkyl quetiapine; MS, microsphere; PLGA, poly(D,L-lactic-co-glycolic acid).

was not observed and the $\mathrm{T}_{\mathrm{g}}$ was observed at about $50^{\circ} \mathrm{C}$. The physical mixture of PLGA 502H and NQ freebase PLGA 502 $\mathrm{H}$ microspheres showed similar results. When the PLGA and the NQ freebase were present together, the $\mathrm{T}_{\mathrm{g}}$ regions were similar, and the difference in the PLGA peaks appeared to be due to the difference in the peak size and ratio.

\section{PXRD}

The PXRD results are shown in Figure 3. A sharp peak was observed at around $10^{\circ}, 15^{\circ}, 20^{\circ}$, and $25^{\circ}$ for NQ. $2 \mathrm{HCl}$, which means that it has the crystallinity of NQ.2HCl. These crystal peaks were not observed for NQ freebase, meaning that the NQ freebase has assumed an amorphous state due to the removal of the base. This implies that the hydrochloride is involved in the crystal structure. ${ }^{22}$ The PLGA $502 \mathrm{H}$, the physical mixture, and the prepared NQ freebase PLGA 502H microspheres were found to be amorphous, which appeared to follow the peak of PLGA 502H. Although the drug is present in the particles and mixture, the peak intensity of the drug is not seen and appears that PLGA 502H peak is dominated. 


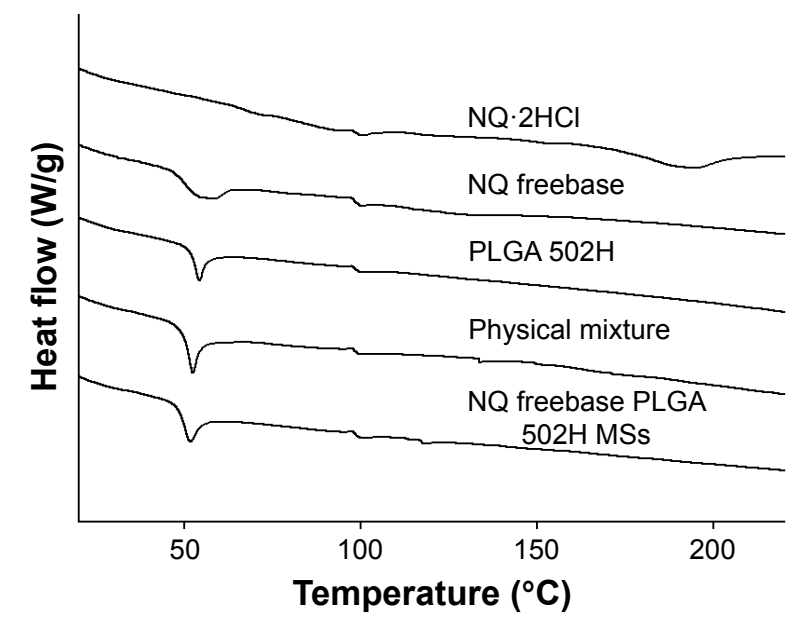

Figure 2 DSC thermograms of NQ freebase PLGA 502H MSs.

Abbreviations: DSC, differential scanning calorimetry; MS, microsphere; NQ, $\mathrm{N}$-desalkyl quetiapine; PLGA, poly(D,L-lactic-co-glycolic acid).

\section{FT-IR}

The FT-IR spectra of NQ·2HCl, NQ freebase, PLGA 502H, NQ freebase PLGA 502H microspheres, and their physical mixture, were obtained and the results are shown in Figure 4. $\mathrm{NQ} \cdot 2 \mathrm{HCl}$ and $\mathrm{NQ}$ freebase showed similar intensity patterns. They had peaks at $1,608 \mathrm{~cm}^{-1}(\mathrm{~N}-\mathrm{H}$ bond $), 928 \mathrm{~cm}^{-1}(\mathrm{C}-\mathrm{H}$ bond), and $765 \mathrm{~cm}^{-1}$ (C-H bond). The peak around $920 \mathrm{~cm}^{-1}$ was only observed for NQ.2HCl. This disappeared for NQ freebase due to the disappearance of the hydrochloride salt as it turned into freebase. The peak of $1,530-1,630 \mathrm{~cm}^{-1}$ for NQ freebase was observed at the peak of the physical mixture, NQ freebase PLGA 502H microspheres. But, they seemed to follow the PLGA 502H peak as a whole. The peak tendency for the NQ freebase PLGA 502H microspheres was similar to

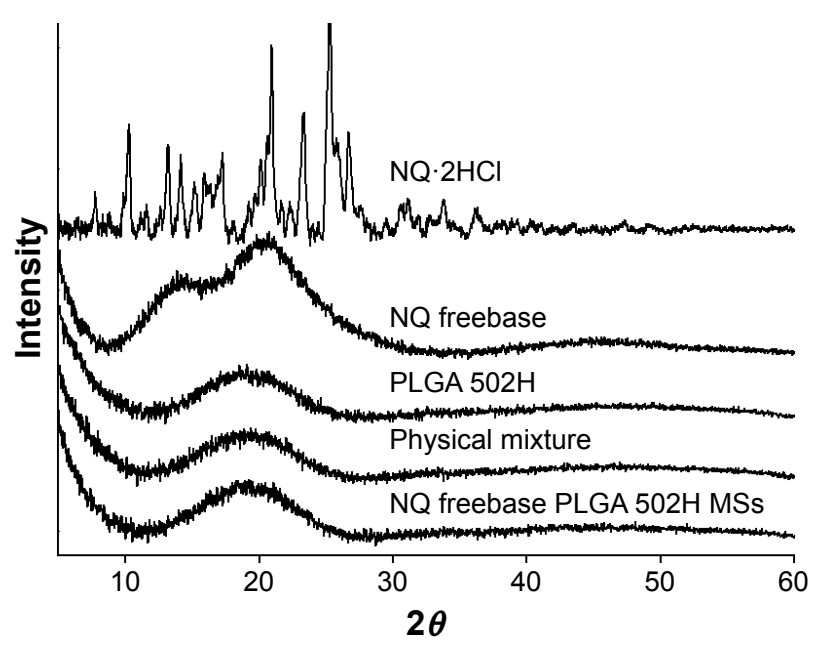

Figure 3 PXRD patterns of NQ freebase PLGA 502H MSs.

Abbreviations: PXRD, powder X-ray diffraction; MS, microsphere; NQ, N-desalkyl quetiapine; PLGA, poly(D,L-lactic-co-glycolic acid).

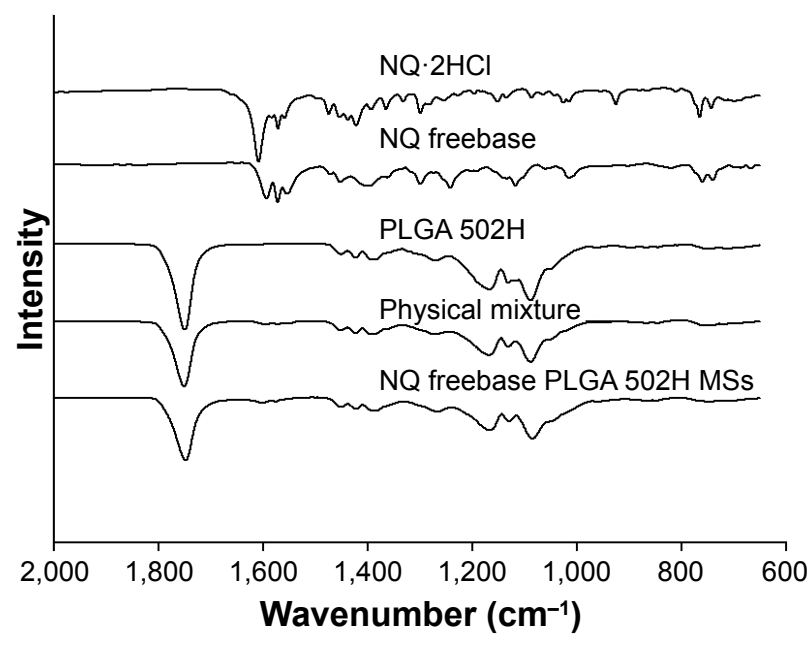

Figure 4 FT-IR spectra of NQ freebase PLGA 502H MSs.

Abbreviations: FT-IR, Fourier-transform infrared spectroscopy; MS, microsphere; $\mathrm{NQ}, \mathrm{N}$-desalkyl quetiapine; PLGA, poly(D,L-lactic-co-glycolic acid).

that of the physical mixture, indicating a tendency dependent upon the ratio between the drug and the polymer. There was no newly formed functional group in the NQ freebase PLGA $502 \mathrm{H}$ microspheres.

\section{In vitro release study}

The dissolution profiles of NQ freebase PLGA 502 microspheres, NQ freebase PLGA 502H microspheres, NQ freebase PLGA 503 microspheres, and NQ freebase PLGA 503H microspheres were obtained, and the results are shown in Figure 5. A typical biphasic release pattern was observed for all formulations, with an initial burst release followed by a more controlled secondary phase. ${ }^{23}$ The rapid release could represent

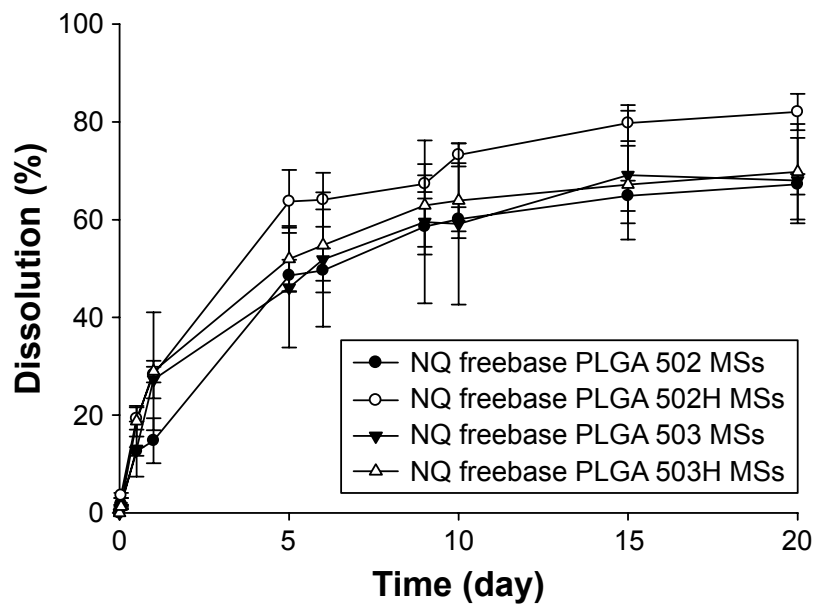

Figure 5 The dissolution patterns of NQ freebase PLGA 502 MSs, NQ freebase PLGA 502H MSs, NQ freebase PLGA 503 MSs, and NQ freebase PLGA 503H MSs (mean $\pm \mathrm{SD}, \mathrm{n}=3$ ).

Abbreviations: MS, microsphere; NQ, N-desalkyl quetiapine; PLGA, poly(D,Llactic-co-glycolic acid); SD, standard deviation. 
the release of poorly entrapped and surface-associated NQ freebase. The drug release during the slower release phase may result from polymer degradation, from drug diffusion through the polymer matrix, or from both. NQ freebase PLGA 502H microspheres released about $20 \%$ of the NQ freebase during the burst-release phase. PLGA 502, PLGA 503, and PLGA $503 \mathrm{H}$ released about $12 \%, 27 \%$, and $29 \%$, respectively, of NQ freebase during the burst release. For 5 days after burst release, $60 \%-70 \%$ of the drug was released. After 5 days, slower dissolution patterns in all formulations were observed. After 20 days, NQ freebase PLGA 502H formulation showed $82.1 \%$ release and other formulations released about $70 \%$ of the drug. The dissolution pattern and entrapment efficiency of the PLGA 502H formulation were suited for the desired 20-day long-lasting formulation. The difference in dissolution patterns would be due to the molecular weight and different terminal group of PLGA, as an increase in molecular weight decreases the dissolution rate and the carboxylic acid terminal catalyzes the hydrolysis of PLGA. Therefore, PLGA 502H microspheres, which have low molecular weight and possess a carboxylic acid terminal group, are shown to have the fastest dissolution pattern. ${ }^{24}$ The release profiles were fitted to various mathematical models to analyze the mechanism of the drug release. The highest correlation coefficient was estimated using the Higuchi matrix kinetics, as shown in Table 3. When plotted in accordance with the Korsmeyer-Peppas method, the $\mathrm{n}$-value was $0.5<\mathrm{n}<1.0$, suggesting non-Fickian transport. Drug diffusion was assumed to be via the dominant mechanism throughout the release period. ${ }^{25}$

\section{In vivo pharmacokinetic study}

Pharmacokinetic profile of NQ freebase solution and NQ freebase PLGA $502 \mathrm{H}$ microspheres following IM administration

During the in vivo test, both groups administered with NQ solution and prepared formulation did not exhibit observable side effects such as rashes, irritation, and abnormal behaviors.

Table 3 Release kinetic profiles of NQ freebase PLGA 502H microspheres

\begin{tabular}{lll}
\hline Model & $\begin{array}{l}\text { Release } \\
\text { constant }(\mathbf{k})\end{array}$ & $\begin{array}{l}\text { Correlation } \\
\text { coefficient }\left(\boldsymbol{R}^{2}\right)\end{array}$ \\
\hline Zero order & 0.7525 & 0.7525 \\
First order & 0.8874 & 0.8874 \\
Higuchi & 4.0433 & 0.9404 \\
Korsmeyer-Peppas & 4.6014 & $0.968 \mathrm{I}$ \\
& $(\mathrm{n}$-value $=0.6083)$ & \\
\hline
\end{tabular}

Abbreviations: NQ, N-desalkyl quetiapine; PLGA, poly(D,L-lactic-co-glycolic acid).

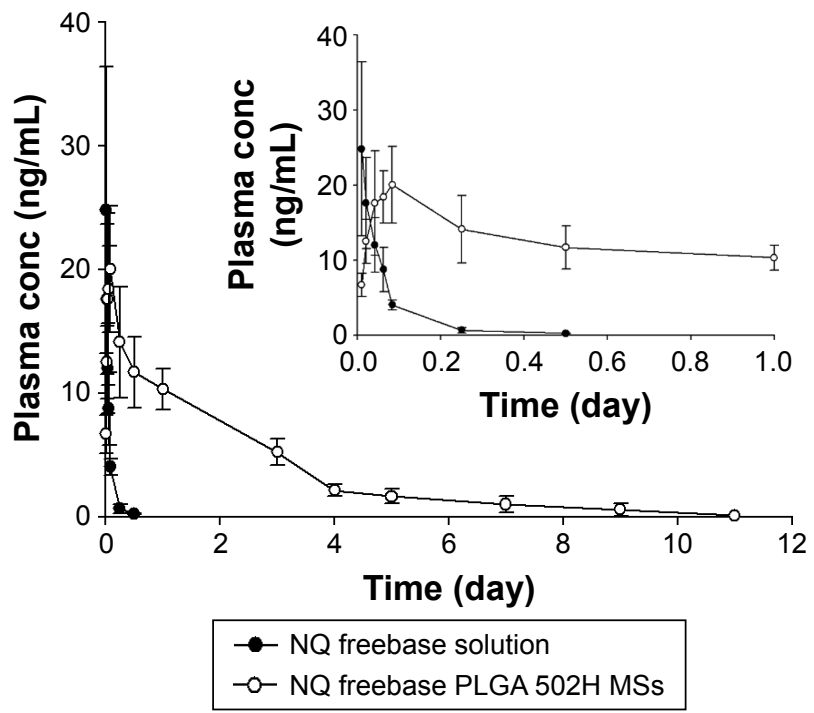

Figure 6 Plasma concentration profile of NQ after intramuscular injection of NQ freebase solution and NQ freebase PLGA 502H MSs (mean \pm SD, $n=6$ ).

Abbreviations: conc, concentration; MS, microsphere; NQ, N-desalkyl quetiapine; PLGA, poly(D,L-lactic-co-glycolic acid); SD, standard deviation.

The mean plasma concentration-time profiles of NQ after IM administration are shown in Figure 6. The calculated pharmacokinetic parameters are shown in Table 4. After administration of the NQ solution, $\mathrm{C}_{\max }$ was observed to be $25.08 \pm 10.41 \mathrm{ng} / \mathrm{mL}$ at time point $0.25 \mathrm{~h}$ (0.01 day). $\mathrm{T}_{\max }$ was observed within $0.25 \mathrm{~h}$ after IM injection. It is presumed that the drug is easily transferred to plasma from muscles. The area under the curve (AUC) was $33.17 \pm 9.33 \mathrm{ng} \cdot \mathrm{h} / \mathrm{mL}$ and $t_{1 / 2}$ was $5.02 \pm 1.28 \mathrm{~h}$. NQ was not observed in plasma after $12 \mathrm{~h}$ of administration. On the other hand, the $\mathrm{C}_{\max }$ of the prepared formulation was observed to be $22.48 \pm 4.46$ $\mathrm{ng} / \mathrm{mL}$, lower than that of the NQ freebase even though 30 times the amount of drug was administered. $t_{1 / 2}$ was $172.79 \pm 23.79 \mathrm{~h}$, which is longer than the half-life of the NQ freebase $(5.05 \pm 1.28 \mathrm{~h})$. The AUC was $916.46 \pm 186.57 \mathrm{ng} \cdot \mathrm{h} /$ $\mathrm{mL}$. The prepared formulation showed $<12$ days of drug release in vivo compared to over 20 days of drug release in

Table 4 Pharmacokinetic parameters of norquetiapine in rat $(n=6)$ administered with NQ freebase solution and NQ freebase PLGA 502H microspheres

\begin{tabular}{lll}
\hline Parameter & $\begin{array}{l}\text { NQ freebase } \\
\text { solution }\end{array}$ & $\begin{array}{l}\text { NQ freebase PLGA } \\
\mathbf{5 0 2 H} \text { microspheres }\end{array}$ \\
\hline $\mathrm{C}_{\max }(\mathrm{ng} / \mathrm{mL})$ & $25.08 \pm \mathrm{II} .4 \mathrm{I}$ & $22.48 \pm 4.46$ \\
$\mathrm{~T}_{\text {max }}(\mathrm{h})$ & $0.29 \pm 0.10$ & $1.50 \pm 0.45$ \\
$\mathrm{t}_{1 / 2}(\mathrm{~h})$ & $5.05 \pm 1.40$ & $172.79 \pm 23.79$ \\
$\mathrm{AUC}(\mathrm{ng} \cdot \mathrm{h} / \mathrm{mL})$ & $33.17 \pm 9.33$ & $916.46 \pm 186.57$ \\
$\mathrm{AUMC}\left(\mathrm{ng} \cdot \mathrm{h}^{2} / \mathrm{mL}\right)$ & $43.40 \pm 9.80$ & $45,421.86 \pm 14,766.68$ \\
\hline
\end{tabular}

Note: Data presented as mean \pm SD.

Abbreviations: AUC, area under the curve; AUMC, area under the moment curve; NQ, N-desalkyl quetiapine; PLGA, poly(D,L-lactic-co-glycolic acid). 


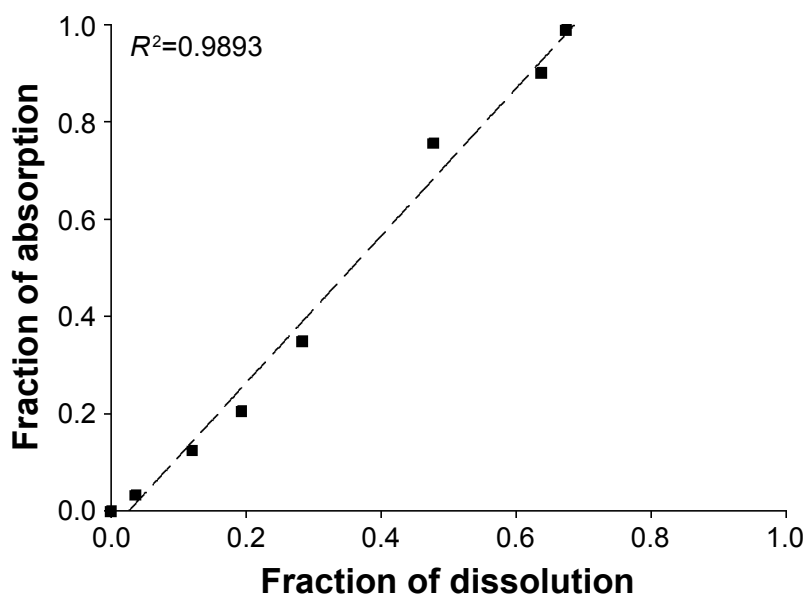

Figure 7 Correlation of the fraction of NQ absorption in vivo versus the fraction of NQ dissolved in vitro.

Abbreviation: NQ, N-desalkyl quetiapine.

vitro. The faster release kinetics in vivo was suggested to be a result of enhanced PLGA degradation, due to the presence of enzymes, limited muscle mass ${ }^{26}$ and other in vivo factors. Similar increased enzymatic polymer degradation in vivo has been reported by other researchers. ${ }^{27,28}$

\section{Correlation of in vitro release and in vivo performance}

The IVIVC allows in vitro study to be used as a surrogate for in vivo study from conventional or long-acting dosage forms. ${ }^{29}$ Level A correlation is the highest level of IVIVC. That represents a point-to-point relationship between in vitro dissolution and in vivo input rate. The same time points (1, $6,12,24,72,120,216 \mathrm{~h}$ ) were chosen, and the fraction of dissolution and fraction of absorption calculated by WagnerNelson method at that point were plotted (Figure 7). The correlation coefficient $\left(r^{2}\right)$ was 0.9893 , indicating a good IVIVC correlation. Although in vivo pharmacokinetic profile was obtained faster than in vitro dissolution profile, it could gain successful correlation in IVIVC. This shows that in vitro drug release test results can be used to predict in vivo performance on mouse models.

\section{Conclusion}

In this study, a sustained release of NQ freebase PLGA microspheres was successfully prepared by o/w emulsionsolvent evaporation method. The physiochemical properties of the synthesized NQ freebase play an important role in the amount of drug loaded in the microspheres. The drug was released from microspheres in PBS medium, following biphasic kinetics. The main mechanism of release was confirmed by the mathematical model to be diffusion and it lasted over 20 days in vitro. The release of the NQ profile of the formulation in vivo was observed to be faster than dissolution, due to various in vivo factors. The long-acting release of the prepared NQ freebase PLGA 502H microspheres compared to that of the NQ freebase solution was shown. These results suggest the potential use of NQ freebase PLGA microspheres for the treatment of schizophrenia over long periods.

\section{Acknowledgment}

This work was supported by the National Research Foundation of Korea (NRF) grant funded by the Korean government (Ministry of Education) (NRF-2015R1D1A1A01059389, NRF-2015R1C1A1A02036702).

\section{Disclosure}

The authors report no conflicts of interest in this work.

\section{References}

1. Higashi K, Medic G, Littlewood KJ, Diez T, Granström O, De Hert M. Medication adherence in schizophrenia: factors influencing adherence and consequences of nonadherence, a systematic literature review. Ther Adv Psychopharmacol. 2013;3(4):200-218.

2. Glazer WM. Review of incidence studies of tardive dyskinesia associated with typical antipsychotics. J Clin Psychiatry. 2000;61 (Suppl 4): $15-20$.

3. López-Muñoz F, Álamo C. Active metabolites as antidepressant drugs: the role of norquetiapine in the mechanism of action of quetiapine in the treatment of mood disorders. Front Psychiatry. 2013;4:102.

4. Leucht S, Corves C, Arbter D, Engel RR, Li C, Davis JM. Secondgeneration versus first-generation antipsychotic drugs for schizophrenia: a meta-analysis. Lancet. 2009;373(9657):31-41.

5. Fura A. Role of pharmacologically active metabolites in drug discovery and development. Drug Discov Today. 2006;11(3):133-142.

6. Bakken GV, Rudberg I, Molden E, Refsum H, Hermann M. Pharmacokinetic variability of quetiapine and the active metabolite N-desalkylquetiapine in psychiatric patients. Ther Drug Monit. 2011; 33(2):222-226.

7. Winter HR, Earley WR, Hamer-Maansson JE, Davis PC, Smith MA. Steady-state pharmacokinetic, safety, and tolerability profiles of quetiapine, norquetiapine, and other quetiapine metabolites in pediatric and adult patients with psychotic disorders. J Child Adolesc Psychopharmacol. 2008;18(1):81-98.

8. Kim D-W, Weon K-Y, Hong E-P, Chung EK, Lee K-T. Comparative physicochemical and pharmacokinetic properties of quetiapine and its active metabolite norquetiapine. Chem Pharm Bull. 2016;64(11): $1546-1554$.

9. Baselt RC, Cravey RH. Disposition of Toxic Drugs and Chemicals in Man. Vol 8. Seal Beach: Biomedical publications; 2011.

10. Castillo EG, Stroup TS. Effectiveness of long-acting injectable antipsychotics: a clinical perspective. Evid Based Mental Health. 2015;18(2): 36-39.

11. Kane JM, Detke HC, Naber D, et al. Olanzapine long-acting injection: a 24-week, randomized, double-blind trial of maintenance treatment in patients with schizophrenia. Am J Psychiatry. 2009;167(2):181-189.

12. Edlund U, Albertsson A-C. Degradable polymer microspheres for controlled drug delivery. Albertsson A-C, editor. Degradable Aliphatic Polyesters. Berlin Heidelberg: Springer-Verlag. 2002:67-112.

13. Burgess DJ, Hickey AJ. Swarbrick J and Boylan JC, editors. Microsphere technology and applications. Encyclopedia of Pharmaceutical Technology. New York: Marcel Dekker Inc., 1994:1-29. 
14. Jain RA. The manufacturing techniques of various drug loaded biodegradable poly (lactide-co-glycolide) (PLGA) devices. Biomaterials. 2000; 21(23):2475-2490.

15. Hickey T, Kreutzer D, Burgess D, Moussy F. Dexamethasone/PLGA microspheres for continuous delivery of an anti-inflammatory drug for implantable medical devices. Biomaterials. 2002;23(7):1649-1656.

16. Guo W, Quan P, Fang L, Cun D, Yang M. Sustained release donepezil loaded PLGA microspheres for injection: preparation, in vitro and in vivo study. Asian J Pharm Sci. 2015;10(5):405-414.

17. Jun H, Lee H-J, Shin B-S, Park C-W. Preparation and in vivo characterization of dual release tablet containing sarpogrelate hydrochloride. J Pharm Invest. 2017;7:1-10.

18. Xu H, Shi Y, Vela S, Marroum P, Gao P. Developing quantitative in vitro-in vivo correlation for fenofibrate immediate-release formulations with the biphasic dissolution-partition test method. J Pharm Sci. 2018;107(1):476-487.

19. O’Donnell PB, McGinity JW. Preparation of microspheres by the solvent evaporation technique. Adv Drug Deliv Rev. 1997;28(1):25-42.

20. Allison SD. Analysis of initial burst in PLGA microparticles. Expert Opin Drug Deliv. 2008;5(6):615-628.

21. Gupta P, Johnson H, Allexon C. In vitro and in vivo evaluation of clarithromycin/poly (lactic acid) microspheres for intramuscular drug delivery. J Control Release. 1993;26(3):229-238.

22. Newman A, Wenslow R. Solid form changes during drug development: good, bad, and ugly case studies. AAPS Open. 2016;2(1):2.
23. Tamaddon L, Mostafavi SA, Karkhane R, Riazi-Esfahani M, DorkooshFA Rafiee-Tehrani M. Design and development of intraocular polymeric implant systems for long-term controlled-release of clindamycin phosphate for toxoplasmic retinochoroiditis. Adv Biomed Res. 2015;4:32.

24. Wischke C, Schwendeman SP. Principles of encapsulating hydrophobic drugs in PLA/PLGA microparticles. Int J Pharm. 2008;364(2): 298-327.

25. Shavi GV, Nayak UY, Reddy MS, et al. A novel long-acting biodegradable depot formulation of anastrozole for breast cancer therapy. Mater Sci Eng C Mater Biol Appl. 2017;75:535-544.

26. Nowland MH. Guidelines on Adjuvant Use in Rabbits Rats and Mice 2016. Available from: https://wiki.med.umich.edu/display/ ULAMGSOP/Guidelines+on+Adjuvant+Use+in+Rabbits+Rats+and+ Mice. Accessed February 28, 2018.

27. Zolnik BS, Burgess DJ. Evaluation of in vivo-in vitro release of dexamethasone from PLGA microspheres. J Control Release. 2008;127(2): 137-145.

28. Sandor M, Harris J, Mathiowitz E. A novel polyethylene depot device for the study of PLGA and P(FASA) microspheres in vitro and in vivo. Biomaterials. 2002;23(22):4413-4423.

29. D'souza SS, DeLuca PP. Methods to assess in vitro drug release from injectable polymeric particulate systems. Pharm Res. 2006;23(3): $460-474$.
Drug Design, Development and Therapy

\section{Publish your work in this journal}

Drug Design, Development and Therapy is an international, peerreviewed open-access journal that spans the spectrum of drug design and development through to clinical applications. Clinical outcomes, patient safety, and programs for the development and effective, safe, and sustained use of medicines are the features of the journal, which

\section{Dovepress}

has also been accepted for indexing on PubMed Central. The manuscript management system is completely online and includes a very quick and fair peer-review system, which is all easy to use. Visit http://www.dovepress.com/testimonials.php to read real quotes from published authors.

Submit your manuscript here: http://www.dovepress.com/drug-design-development-and-therapy-journal 